\title{
Quantum Encodings in Spin Systems and Harmonic Oscillators
}

\author{
Stephen D. Bartlett,, 月 Hubert de Guise, ${ }^{1,2}$ and Barry C. Sanders ${ }^{1,3}$ \\ ${ }^{1}$ Department of Physics, Macquarie University, Sydney, New South Wales 2109, Australia \\ ${ }^{2}$ Department of Physics, Lakehead University, Thunder Bay, Ontario, P7B 5E1, Canada \\ ${ }^{3}$ Erwin Schrödinger International Institute for Mathematical Physics, Boltzmanngasse 9, A-1090 Vienna, Austria
}

(Dated: April 12, 2002)

\begin{abstract}
We show that higher-dimensional versions of qubits, or qudits, can be encoded into spin systems and into harmonic oscillators, yielding important advantages for quantum computation. Whereas qubit-based quantum computation is adequate for analyses of quantum vs classical computation, in practice qubits are often realized in higher-dimensional systems by truncating all but two levels, thereby reducing the size of the precious Hilbert space. We develop natural qudit gates for universal quantum computation, and exploit the entire accessible Hilbert space. Mathematically, we give representations of the generalized Pauli group for qudits in coupled spin systems and harmonic oscillators, and include analyses of the qubit and the infinite-dimensional limits.
\end{abstract}

PACS numbers: 03.67.Lx, 02.20.-a, 42.50.-p

Quantum computation may be able to perform certain tasks more efficiently than a classical computer; for example, Shor's algorithm [1] for factoring prime numbers on a quantum computer is exponentially faster than any known algorithm on a classical computer. The standard model of a quantum computer involves coupling together two-level quantum systems (qubits) such that the Hilbert space of the system grows exponentially in the number of qubits.

A major obstacle to universal quantum computing is the limit on the number of coupled qubits that can be achieved in a physical system [2]. The use of $d-$ dimensional, or qudit, quantum computing enables a much more compact and efficient information encoding than for qubit computing. Qudit quantum information processing employs fewer coupled quantum systems: a considerable advantage for the experimental realization of quantum computing. The harmonic oscillator is a system that naturally provides qudits as quanta in its energy spectrum. Qubits are obtained by restricting the dynamics to just two of these quanta, namely the vacuum state $|0\rangle$ and the first excited state $|1\rangle$; e.g., photons in cavity QED [3] and interferometry [4]. However, the control of entanglement in larger Hilbert spaces is now feasible (e.g., orbital angular momentum states of photons [5]). Our aim is to show that the restriction to two-dimensional Hilbert spaces is not necessary and that higher-dimensional Hilbert spaces are an advantage, particularly when the number of achievable coupled systems is limited and entanglement between systems with larger Hilbert spaces is physically possible.

A quantum computer also requires gates, realized as the unitary evolution under some Hamiltonian. For qubits, a universal set of gates is given by arbitrary $\mathrm{SU}(2)$ rotations of a single qubit along with some nonlinear coupling transformation between adjacent qubits generated by a two-qubit Hamiltonian [6]. For qudit quantum computation, the issue of creating a universal set of gates is more involved. In particular, it is not possible to treat coupled qudits as a collection of qubits, because (typically) one does not have access to "pairwise" Hamiltonians between two arbitrary levels of coupled qudits. For example, in a system of coupled oscillators realized as radiation modes in a cavity, Hamiltonians that generate single-gate operations such as a coupling of the $i^{\text {th }}$ level of one oscillator and the $j^{\text {th }}$ level of another cannot be realized physically. Thus, quantum computation with qudits requires an investigation not only into the coupling of multilevel systems but also the set of physically realizable Hamiltonians with which one can construct a universal set of gates. In this communication, we develop transformations for a collection of coupled $d-$ level systems. These transformations are obtained as two mathematical realizations of a basis of unitary operators for a single qudit. We show how each of these realizations can be implemented either in a spin system or a harmonic oscillator [17]. We establish a SUM gate [7, which couples qudits and serves as the qudit analogue of the controlled NOT gate; this SUM gate employs a standard two-mode coupling Hamiltonian.

The theoretical investigation of qudit computation is best expressed in terms of the generalized Pauli group for qudits. Recalling the Pauli group for a two-level system, a qubit is realized as a state in a two-dimensional Hilbert space $\mathcal{H}_{2}$, spanned by two normalized orthogonal states, $|0\rangle$ and $|1\rangle$, that serve as a computational basis for $\mathcal{H}_{2}$. The unitary operators $\left\{X_{2} \equiv \sigma_{x}, Z_{2} \equiv \sigma_{z}\right\}$, where $\sigma_{i}$ is a Pauli spin matrix, generate the Pauli group using matrix multiplication: the elements of this group are known as Pauli operators and provide a basis of unitary operators on $\mathcal{H}_{2}$.

A qudit is realized as a state in a $d$-dimensional Hilbert space $\mathcal{H}_{d}$, with a computational basis $\{|s\rangle ; s=$ $0,1, \ldots, d-1\}$ serving as the generalization of the binary basis $\{|0\rangle,|1\rangle\}$ of the qubit. A basis for unitary operators 
on $\mathcal{H}_{d}$ is given by the generalized Pauli operators [7, 8]

$$
\left(X_{d}\right)^{a}\left(Z_{d}\right)^{b}, \quad a, b \in 0,1, \ldots d-1,
$$

where $X_{d}$ and $Z_{d}$ are defined by their action on the computational basis as follows:

$$
\begin{aligned}
X_{d}|s\rangle & =|s+1(\bmod d)\rangle, \\
Z_{d}|s\rangle & =\exp (2 \pi \mathrm{i} s / d)|s\rangle .
\end{aligned}
$$

The operators $X_{d}$ and $Z_{d}$ generate the noncommutative generalized Pauli group under matrix multiplication, satisfying

$$
Z_{d} X_{d}=\exp (2 \pi \mathrm{i} / d) X_{d} Z_{d} .
$$

The analysis of the generalized Pauli group as operators in spin systems and harmonic oscillators is necessary for realizing qudit algorithms and error correcting codes [7, 9]. For spin systems, we construct the generators of the generalized Pauli group in $d=2 j+1$ dimensions using operators that are expressed in terms of the $\mathrm{SU}(2)$ angular momentum and phase operators. This construction allows us to conveniently view a qudit as the Hilbert space of a $d$-dimensional irreducible representation (irrep) of $\mathrm{SU}(2)$. For qudits in a harmonic oscillator, we obtain a generalized Pauli group generated by the number operator $\hat{N}$ and a phase operator $\hat{\theta}$. A second realization of qudits is given in terms of phase states; this realization is "dual" to the first realization given here, and allows for the construction of a simple SUM gate. By investigating the $d \rightarrow \infty$ limit, we show that it is not the common generalization of the Pauli group for continuous-variable quantum information (i.e., the Heisenberg-Weyl group) with position eigenstates as the computational basis.

We begin by constructing a realization of the generalized Pauli group for a spin system; i.e., in the $d-$ dimensional Hilbert space of a $\mathrm{SU}(2)$ irrep of highest weight (angular momentum) $j=(d-1) / 2$. Consider the standard basis for the $\mathrm{su}(2)$ algebra $\left\{\hat{J}_{z}, \hat{J}_{ \pm}=\hat{J}_{x} \pm \mathrm{i} \hat{J}_{y}\right\}$. Let $\left.\{\mid j, m)_{z} ; m=-j, \ldots, j\right\}$ denote the standard weight basis for the Hilbert space $\mathcal{H}_{d=2 j+1}$ for an $\mathrm{SU}(2)$ irrep of highest weight (angular momentum) $j$. We use a simplifying notation, allowing $m$ to take all the integer (or half-integer) values modulo $2 j+1$, thus defining $\left.\mid j, j+1)_{z}=\mid j,-j\right)_{z}$.

With the computational basis defined to be

$$
|s\rangle \equiv \mid j, j-s)_{z}, \quad s=0,1, \ldots, d-1,
$$

the generators of the generalized Pauli group can be expressed in terms of operators that act in a natural way on $\mathrm{SU}(2)$ basis states. Because the basis states are eigenstates of $\hat{J}_{z}$, we have

$$
\begin{aligned}
X_{d} & \left.\mapsto \sum_{m=-j}^{j} \mid j, m\right)_{z}(j, m+1 \mid, \\
Z_{d} & \mapsto \exp \left(2 \pi \mathrm{i}\left(j-\hat{J}_{z}\right) / d\right),
\end{aligned}
$$

which are unitary and satisfy Eqs. (2014).

The operators $X_{d}$ and $Z_{d}$ are conjugate to one another:

$$
X_{d}=U^{-1} \cdot Z_{d} \cdot U
$$

where the unitary transformation $U$ is the Fourier transform in dimension $d$. It is convenient to view $X_{d}$ as the exponent of a Hermitian operator $\hat{\theta}_{z}$, defined so that $X_{d}=\exp \left(2 \pi \mathrm{i} \hat{\theta}_{z} / d\right)$, just as $Z_{d}$ is generated by the operator $\hat{J}_{z}$. The operator $\hat{\theta}_{z}$ is known as a phase operator 10] for a spin system.

The generalized Pauli operators $X_{d}$ and $Z_{d}$ can also be realized as operators that act naturally on the space $\mathbb{H}_{d}$ of dimension $d$ spanned by harmonic oscillator states of no more than $d-1$ bosons. We define the computational basis to be the set of harmonic oscillator energy eigenstates

$$
|s\rangle \equiv|n=s\rangle_{\mathrm{HO}}, \quad s=0,1, \ldots, d-1,
$$

where $\hat{N}|n\rangle_{\mathrm{HO}}=n|n\rangle_{\mathrm{HO}}$. Again, we apply the cyclic notation $|d\rangle=|0\rangle$. Now defining the generalized Pauli group as operators on this subspace of the harmonic oscillator, the generators $X_{d}$ and $Z_{d}$ are expressed as

$$
X_{d} \mapsto \sum_{s=0}^{d-1}|s+1\rangle\langle s|, \quad Z_{d} \mapsto \exp (2 \pi \mathrm{i} \hat{N} / d),
$$

which are unitary on $\mathbb{H}_{d}$. Again, we view $X_{d}$ as the exponent of a Hermitian operator $\hat{\theta}_{z}$, such that $X_{d}=$ $\exp \left(2 \pi \mathrm{i} \hat{\theta}_{z} / d\right)$; the operator $\hat{\theta}_{z}$ is the Pegg-Barnett phase operator [11], which is well-defined for finite $d$. We will call this representation of the generalized Pauli group the number representation.

An advantage of this explicit realization of $X_{d}$ and $Z_{d}$ as unitary operators on the harmonic oscillator Hilbert space is that it enables us to explore the $d \rightarrow \infty$ limit in a rigorous way; this limit yields continuous-variable quantum computation. The limiting procedure for phase operators has been thoroughly investigated [11, 12]. In this limit, the computational basis remains the harmonic oscillator energy eigenstates (now including all states $s=$ $0,1, \ldots, \infty)$, following Eq. (9). Note that defining the phase operator on the infinite-dimensional Hilbert space $\mathbb{H}_{\infty}$ of the harmonic oscillator presents challenges [12]. The number operator $\hat{N}$ and the phase operator $\hat{\theta}_{z}$ are conjugate in the same sense that momentum and position are conjugate, but the limit does not yield the usual continuous-variable Pauli group: the HeisenbergWeyl group, with position $\hat{x}$ and momentum $\hat{p}$ operators as generators. It is also important that the states of the computational basis for the limiting case remain harmonic oscillator energy eigenstates, rather than position (or momentum) eigenstates or squeezed Gaussians, as are commonly used for continuous-variable quantum computing. 
A second realization of $X_{d}$ and $Z_{d}$ in the Hilbert space $\mathcal{H}_{d}$ for an irrep of $\mathrm{SU}(2)$ can be constructed, with a computational basis given by $\mathrm{SU}(2)$ phase states; this representation is "dual" to the number representation. Consider the relation $\mathrm{i} X_{2}=\exp \left(\mathrm{i}(\pi / 2) X_{2}\right)$ for qubits; i.e., that

$$
|1\rangle=X_{2}|0\rangle=(-\mathrm{i}) e^{\mathrm{i}(\pi / 2) X_{2}}|0\rangle .
$$

The Pauli operator $X_{2}$ has two interpretations, each of which can be generalized in a different way. In the number representation, we interpret $X_{2}$ as a cyclic number state raising operator $|1\rangle=X_{2}|0\rangle$ and generalize this operator as a cyclic raising operator. However, using the relation (11), we can also view $X_{2}$ as a rotation. (Using the $\mathrm{su}(2)$ representation $X_{2}=2 \hat{J}_{x}$, this rotation is about the $x$-axis.) Thus, the state $|1\rangle$ is obtained (up to a phase) by rotating $|0\rangle$ by an angle $\pi$ about the $x$-axis. The computational basis states needed for this type of generalization to qudits are $\mathrm{SU}(2)$ phase states and have been investigated by Vourdas 10 (although using rotations generated by $\hat{J}_{z}$ rather than $\left.\hat{J}_{x}\right)$. These states form an orthonormal basis for the $\mathrm{SU}(2)$ irrep.

Let $\left.\{\mid j, m)_{x} ; m=-j, \ldots, j\right\}$ be the weight basis for an $\mathrm{SU}(2)$ irrep of angular momentum $j=(d-1) / 2$, where $\hat{J}_{x}$ rather than $\hat{J}_{z}$ is diagonal; i.e., $\left.\left.\hat{J}_{x} \mid j, m\right)_{x}=m \mid j, m\right)_{x}$. For this representation, we define the computational basis states to be

$$
|s\rangle \equiv \begin{cases}\left.\frac{1}{\sqrt{d}} \sum_{m=-j}^{j} \exp (2 \pi \mathrm{i} m s / d) \mid j, m\right)_{x} & d \text { odd }, \\ \left.\frac{1}{\sqrt{d}} \sum_{m=-j}^{j} \exp \left(2 \pi \mathrm{i}\left(m+\frac{1}{2}\right) s / d\right) \mid j, m\right)_{x} & d \text { even. }\end{cases}
$$

These states form an orthonormal basis for $\mathcal{H}_{d}$ 10]. They are referred to as $\mathrm{SU}(2)$ phase states because they are eigenstates of a phase operator for spin systems.

The generalized Pauli operator $X_{d}$ on this computational basis is given by

$$
X_{d} \mapsto \begin{cases}\exp \left(2 \pi \mathrm{i} \hat{J}_{x} / d\right) & d \text { odd } \\ \exp (-\mathrm{i} \pi / d) \exp \left(2 \pi \mathrm{i} \hat{J}_{x} / d\right) & d \text { even }\end{cases}
$$

satisfying Eq. (2). Note that $\left(X_{d}\right)^{d}=\hat{\mathbb{1}}$ for both $j$ integral and half-integral. The generalized Pauli operator $Z_{d}$ is given by

$$
Z_{d} \mapsto \sum_{s=0}^{d-1} \exp (2 \pi \mathrm{i} s / d)|s\rangle\langle s|,
$$

which is unitary and satisfies Eq. (3). Note that we can express $Z_{d}$ as the exponent of a Hermitian operator,

$$
Z_{d}=\exp \left(2 \pi \mathrm{i} \hat{\theta}_{x} / d\right), \quad \hat{\theta}_{x} \equiv \sum_{s=0}^{d-1} s|s\rangle\langle s| ;
$$

the operator $\hat{\theta}_{x}$ is a phase operator for a spin system.
Note that this representation of the generalized Pauli group is "dual" to the number representation of Eqs. (6)(7) in the same sense that the position and momentum representations of the harmonic oscillator are dual. For the number representation, the computational basis states are eigenstates of $\hat{J}_{z}$, and the phase operator $\hat{\theta}_{z}$ generates the "ladder" transformations. In the phase representation given here, the computational basis states are eigenstates of the phase operator $\hat{\theta}_{x}$, i.e., "phase eigenstates", and it is $\hat{J}_{x}$ which generates the ladder transformations via rotations about the $x$-axis. Both of these representations can be considered as natural generalizations of the qubit case, because the standard computational basis $\left.|0\rangle=\mid \frac{1}{2}, \frac{1}{2}\right)_{z}$ and $\left.|1\rangle=\mid \frac{1}{2},-\frac{1}{2}\right)_{z}$ are both eigenstates of $\hat{J}_{z}$ and phase eigenstates of $\hat{\theta}_{x}$.

As with the number representation, this phase representation of the generalized Pauli group can be expressed in a harmonic oscillator Hilbert space. Again considering the finite Hilbert space $\mathbb{H}_{d}$, the eigenstates of $\hat{J}_{x}$ (unlike $\hat{J}_{z}$ of the number representation) are replaced with harmonic oscillator number states $|n\rangle$ with a boson number less than $d$. The computational basis, then, consists of finite- $d$ phase eigenstates. The generalized Pauli operators $X_{d}$ and $Z_{d}$ are generated by the number operator and Pegg-Barnett phase operator, respectively. Again, the $d \rightarrow \infty$ limit yields challenging problems: it is well known that phase eigenstates do not exist in the infinitedimensional Hilbert space $\mathbb{H}_{\infty}$ of the harmonic oscillator [11].

Despite the issues involving $d \rightarrow \infty$ phase operators, universal qudit quantum computation is well-defined for finite $d[7]$. In the following, we discuss these requirements in terms of an optical realization, where the harmonic oscillators are realized as modes in a cavity; such a realization has been discussed in 13. However, this realization is formally equivalent to any oscillator system.

To perform arbitrary unitary transformations on a single oscillator efficiently, one may employ a combination of linear optics, squeezing, and a nonlinear process such as photon detection [f] or a nonlinear optical Kerr interaction [14. Of particular importance is to realize the Fourier transform operation on a single qudit, which takes number eigenstates to phase eigenstates and vice versa. This operation is the generalization of the Hadamard transformation for qubits; as it is a unitary transformation on a single oscillator, it can be performed efficiently as described above.

For quantum computation, we must also realize a gate that performs a two-qudit interaction. A simple controlled two-qudit interaction gate is the SUM gate [7]

$$
\mathrm{SUM}:\left|s_{1}\right\rangle_{1} \otimes\left|s_{2}\right\rangle_{2} \mapsto\left|s_{1}\right\rangle_{1} \otimes\left|s_{1}+s_{2}(\bmod d)\right\rangle_{2} .
$$

Consider two oscillators coupled by the four-wave mixing interaction Hamiltonian $\chi \hat{N}_{1} \hat{N}_{2}=\chi \hat{a}_{1}^{\dagger} \hat{a}_{1} \hat{a}_{2}^{\dagger} \hat{a}_{2}$. This 
Hamiltonian for an optical system describes a four-wave mixing process in which $\chi$ is proportional to the thirdorder nonlinear susceptibility 15. Let oscillator 1 be in a state $\left|s_{1}\right\rangle_{1}$ encoded in the number state basis, and let oscillator 2 be in a state $\left|s_{2}\right\rangle_{2}$ encoded in the phase state basis. This interaction Hamiltonian generates the transformation

$e^{-\mathrm{i} \chi \hat{N}_{1} \hat{N}_{2} t}\left|s_{1}\right\rangle_{1} \otimes\left|s_{2}\right\rangle_{2}=\left|s_{1}\right\rangle_{1} \otimes\left|\left(\frac{\chi t}{2 \pi}\right) s_{1}+s_{2}(\bmod d)\right\rangle_{2}$.

Thus, with fixed interaction time $t=2 \pi \chi^{-1}$, this Hamiltonian generates the SUM transformation on two qudits. (Note that a similar gate can be defined for spin systems using a $\hat{J}_{z 1} \hat{J}_{z 2}$-type Hamiltonian [16.)

Quantum computation with multiple qudits could be performed by coupling several modes in a single cavity; each mode realizes a single qudit 13]. Modes are coupled via a SUM interaction of the time described above. Note that the control qudit for the sum operation must be encoded in the number state basis, and the target qudit must be in the phase state basis. The encodings of each qudit can be swapped (between number and phase state bases) using the Fourier transform.

In summary, we have presented realizations of qudit quantum computation in spin systems and harmonic oscillators in terms of number and phase operators. The representations of the generalized Pauli group, viewed in terms of $\mathrm{SU}(2)$ or harmonic oscillator operators, allows for qudits to be explicitly encoded into such systems. An advantage of this scheme is that the SUM gate employs a standard two-mode Hamiltonian to couple two qudits. From a rigorous mathematical viewpoint, these realizations give natural extensions of the qubit-based Pauli group, and allow for the investigation of the $d \rightarrow \infty$ limit and continuous-variable quantum computation.

By employing qudits rather that qubits, the full size of the accessible Hilbert space can be exploited, with the advantage of requiring fewer coupled systems for a given quantum information process. However, the use of qudits requires a different set of quantum gates than the usual qubit rotations and two-qubit interactions that are normally assumed. The realization of a universal set of gates using linear optics, squeezing, and a nonlinear interaction is convenient for certain harmonic oscillator systems but is not unique; an important challenge is to identify the optimal set of gates for a particular system. The analysis presented here provides the necessary theoretical tools for developing qudit quantum computation in spin systems and harmonic oscillators as a promising alternative to qubit quantum computation.

This project has been supported by an Australian Research Council Large Grant and by a Macquarie University Research Grant. SDB acknowledges the support of a Macquarie University Research Fellowship. We acknowledge helpful discussions with S. L. Braunstein, T. Rudolph and B. T. H. Varcoe.

* Electronic address: bartlett@ics.mq.edu.au

[1] P. W. Shor, Proceedings, $35^{\text {th }}$ Annual Symposium on Foundations of Computer Science (IEEE Press, Los Alamitos, CA, 1994).

[2] A. Steane, Rept. Prog. Phys. 61, 117 (1998).

[3] V. Giovannetti, D. Vitali, P. Tombesi and A. Ekert, Phys. Rev. A 62, 032306 (2000).

[4] E. Knill, R. Laflamme, and G. J. Milburn, Nature (London) 409, 46 (2001).

[5] A. Mair, A. Vaziri, G. Weihs, and A. Zeilinger, Nature (London) 412, 313 (2001).

[6] M. A. Nielsen and I. L. Chuang, Quantum Computation and Quantum Information (Cambridge University Press, Cambridge, 2000).

[7] D. Gottesman, A. Kitaev and J. Preskill, Phys. Rev. A 64, 012310 (2001).

[8] J. Patera and H. Zassenhaus, J. Math. Phys. 29, 665 (1988).

[9] D. Gottesman, "Fault-Tolerant Quantum Computation with Higher-Dimensional Systems," quant-ph/9802007.

[10] A. Vourdas, Phys. Rev. A 41, 1653 (1990).

[11] D. T. Pegg and S. M. Barnett, J. Mod. Optics 44, 225 (1997).

[12] R. Lynch, Phys. Rep. 256, 368 (1995).

[13] S. D. Bartlett, B. C. Sanders, B. T. H. Varcoe and H. de Guise, in Experimental Implementation of Quantum Computation (IQC'01) ed. R. Clark, (Rinton, Princeton, NJ, 2001), pp. 344-347.

[14] S. Lloyd and S. L. Braunstein, Phys. Rev. Lett. 82, 1784 (1999).

[15] G. J. Milburn and D. F. Walls, Phys. Rev. A 28, 2065 (1983).

[16] B. C. Sanders, Phys. Rev. A 40, 2417 (1989).

[17] An inequivalent encoding of a qudit in a harmonic oscillator is given in []]. 